\title{
JUSTIFICACIÓN DE LOS "DERECHOS HUMANOS"
}

AGUSTÍN PÉREZ CARRILLO

UNIVERSIDAD AUTÓNOMA METROPOLTTANA

\section{Algunas ideas sobre el concepto de justificación}

En diversas ocasiones he apuntado una expresión de Wittgenstein según la cual la justificación implica apelar a algo independiente. Toda justificación, desde esta perspectiva, se presenta en una relación entre dos términos: lo que se justifica y lo que justifica.

"Lo que se justifica" puede tener diversos estados ontológicos, así sea una decisión, una norma, una operación matemática, una acción, un argumento, una elección, un enunciado, etc., e indica en gran medida que no es posible que se justifique por sí y en sí en virtud de algunas notas, propiedades o características que le sean inherentes.

"Lo que justifica" ha de cumplir algunos requerimientos, de acuerdo con el universo de discurso en el cual se plantean las exigencias de justificación, para no aparecer como dogmático o arbitrario.

Ser algo independiente como parte del concepto de justificación puede entenderse en dos proyecciones: no depender de lo que se justifica ni requerir de una instancia que lo justifique en el universo de discurso previamente señalado. Así, se rechazan la autojustificación y las justificaciones sin límite.

Un uso de la palabra "justificación" proviene del latín iustificare equivalente a "hacer justo a uno" y de allí a probar o demostrar; la palabra "justo" deriva de iustus con el significado de "conforme a la justicia y la razón". En este último sentido se advierten los dos términos de la relación en virtud del empleo de la palabra "conforme" entre distintas variables.

Existen conceptos próximos al significado de la palabra "justificación"; así, "verificación", "demostración", "prueba", "deducción", "fundamentación", "derivación" y otras. Posiblemente cada uno de ellos tenga una mayor aplicación y reconocimiento en algunas disciplinas en particular; así, el término "verificación" se utiliza en las ciencias naturales y alude, en principio, a la búsqueda de la verdad de los enunciados descriptivos mediante la comparación de éstos con la "realidad". Los conceptos de "demostración", 
"prueba”, "derivación" y "deducción" son más utilizados en el lenguaje de las ciencias formales como la lógica, las matemáticas y la geometría. Así, se dice, se demuestra un teorema, se deduce o deriva una conclusión. La palabra "fundamentación" tiene un amplio uso en el derecho y en la política y en las disciplinas que los estudian: la ciencia jurídica y la politología, respectivamente. Es normal aludir a la fundamentación constitucional de los actos de las autoridades en tanto se afirma su conformidad con los preceptos del máximo ordenamiento jurídico. En política se habla de los fundamentos de la democracia como sistema de gobierno al aludirse a la libertad de expresar argumentos, a la tolerancia y a la posibilidad real de obtener compromisos.

En relación con las creencias empíricas, sostiene C.I. Lewis, existen dos dimensiones importantes: su verificación y su justificación; la determinación de la creencia como verdadera y la determinación de la creencia como racionalmente creíble. Un enunciado es analizable con base en estas exigencias y así resulta que puede ser: verificado y justificado; no verificado y justificado; verificado y no justificado, y no verificado ni justificado. Estas combinaciones reflejan las posibilidades para calificar los enunciados y representan la idea de que, aun cuando un enunciado sea verdadero, en la proyección científica se requiere su justificación para tenerlo por validado y no tildarlo de arbitrario o casual. ${ }^{1}$

Las preguntas acerca de la justificación de algo, un enunciado por ejemplo, coadyuvan a determinar el ámbito en el cual ha de producirse la respuesta. Sin embargo, pueden surgir nuevas preguntas con el ánimo de encontrar mejores justificaciones en cada una de las respuestas, debido a los intereses de diversa índole presentes en la empresa del conocimiento y de la acción humana.

En todos los ejemplos aludidos el común denominador es la existencia de aquello que se pretende justificar y de la instancia independiente, lo que justifica, con la cual se pretende hacer la justificación, esto es, establecer una relación.

En el parágrafo 265 de las Investigaciones filosóficas, Wittgenstein sostiene: "Mediante un diccionario se puede justificar la traducción de una palabra $X$ por una palabra $Y$. ¿Pero debemos también decir que se trata de una justificación cuando esa tabla sólo se consulta de la imaginación? Bueno, entonces es precisamente una justificación subjetiva [...] Pero la justificación consiste, por cierto, en apelar a una instancia independiente.

1 C.I. Lewis, An Analysis of Knowledge and Valuation, 4a. reimpresión, Foundation Established in Memory of Paul Carus, 1852-1919, La Salle (Ill.), 1971, p. 254 ss. 
Consultar una tabla en la imaginación es tan poco consultar una tabla, como la imagen del resultado de un experimento imaginado es el resultado de un experimento." 2

Además, indica en $\S 320$, se puede estar seguro de realizar una actividad y a esta seguridad se la llama "bien fundada": "si, por ejemplo, se me da una función algebraica, estoy SEGURO de poder completar sus valores para los argumentos 1, 2, 3, hasta 10. Esta seguridad se llama 'bien fundada', pues he aprendido a computar estas funciones, etc. En otros casos no estará fundada -pero estará justificada, sin embargo, por el éxito."3 Además se puede tener la confianza de que las cosas serán de cierta manera en virtud del "sistema de hipótesis, de leyes naturales, en que consideramos el fenómeno de la certeza". ${ }^{4}$ En última instancia, como la justificación forma juegos de lenguaje, que la confianza esté justificada dependerá de lo que admiten como justificación, a través del lenguaje, los seres humanos en las diversas formas de vida. La regularidad en el juego de las justificaciones tiene un papel central en el éxito. Fundar es una de las formas de justificar. Así, si de $P \&-P$ derivo $Q$, la derivación está fundada en las reglas de la lógica formal y, por ende, está justificada. Un enunciado no está fundado en la inducción, y la confianza se justifica por el éxito que se ha tenido. ${ }^{5}$

En lógica matemática elemental se justifican los argumentos de acuerdo con el sistema de definiciones, reglas y proposiciones determinadas. Una de esas reglas señala: "un argumento es correcto (válido) si su conclusión se sigue de o es una consecuencia de sus premisas; de otro modo es incorrecto", o, en otras palabras: "un argumento es correcto si y solamente si no es posible que sus premisas sean verdaderas y su conclusión falsa". ${ }^{6}$

La calificación de correcto o incorrecto para un argumento se justificará de acuerdo con la definición ofrecida. "Algunos hombres son inteligentes; algunos primates son hombres; por consiguiente, algunos primates son inteligentes" y "Algunos profesores son suecos; algunos noruegos son profesores; por consiguiente, algunos noruegos son suecos" (Benson Mates, Lógica matemática elemental, pp. 17-19). Los dos argumentos son incorrectos, esto es, no están justificados en el sistema de lógica matemática elemental. En cambio "Todos los hombres son inteligentes; todos los primates son hombres; por consiguiente, todos los primates son inteligentes",

2 Ludwig Wittgenstein, Investigaciones filosóficas, trad. de Ulises Moulines, Instituto de Investigaciones Filosóficas, Universidad Nacional Autónoma de México/Editorial Crítica/Grupo Editorial Grijalbo, Barcelona/México, 1986.

3 Ludwig Wittgenstein, op. cit., § 320.

4 Ibid., $\S 325$.

5 Ibid., $\$ 324$.

6 Benson Mates, Lógica matemática elemental, Editorial Tecnos, Madrid, 1971, p. 18 ss. 
es un argumento correcto y se justifica en el sistema de lógica matemática elemental. ${ }^{7}$

Sissela Bok sostiene que "justificar es defender algo como justo, correcto o propio, proporcionando razones adecuadas; implica sostener algún estándar religioso, legal o moral". Tal justificación, según la misma autora, requiere un público, la cual puede ser directamente Dios, o una corte legal, o nuestros clientes o nuestra conciencia; aunque en ética es más apropiado intentar la referencia no a cualquier individuo o público, sino más bien "a las personas razonables en general”. La justificación moral, en consecuencia, no puede ser exclusiva u oculta; tiene que ser susceptible de publicarse. $\mathrm{Al}$ ir más lejos de lo privado, intenta trascender también de lo meramente subjetivo. $^{8}$

¿Qué puede significar en el pensamiento de Sissela Bok que la justificación se hace ante nuestra conciencia? En primer término, que hay una instancia en nosotros mismos con ciertas características, calidad y capacidad para comprender las razones de una acción o de una omisión y, en su caso, aceptarlas y hasta aprobarlas.

Dar cuenta, responder nosotros mismos ante la parte denominada conciencia, puede ser un estado mental y como tal de dificil, si no de imposible, verificación, aun cuando puede hacerse algún tipo de referencia a ella a través de otros hechos. De acuerdo con algunas teorías, esta instancia psíquica se hace patente y se presenta en las situaciones problemáticas de carácter moral y religioso.

Para un entendimiento posible de esta problemática se pueden presentar las teorías de Freud sobre el superyó y de Tomás de Aquino en relación con la sindéresis. Explicarlas no supone acuerdo con alguna de ellas sino simplemente un recurso para comprender formas de justificar las acciones.

El tema de la justificación ha sido una preocupación constante en filosofía y en diversas disciplinas; generalmente quien se ocupa de esta problemática alude a la búsqueda de instancias independientes. "Los filósofos, sostiene Rawls, tratan de justificar las teorías éticas en una de dos formas. A veces intentan encontrar principios autoevidentes, de los que pueda derivarse un cuerpo suficiente de normas y preceptos para explicar nuestros juicios. Podemos considerar una justificación de este tipo como cartesiana. Presupone que los primeros principios pueden ser apreciados como verdaderos, $\mathrm{e}$ incluso tienen que serlo, necesariamente; después, el razonamiento deductivo traslada esta convicción de las premisas a la conclusión. Una segunda actitud (llamada naturalismo, por un abuso del lenguaje) consiste en introducir

7 Benson Mates, op. cit.

8 Sissela Bok, Lying. Moral Choice in Public and Private Life, Vintage Books, A divisional of Random House, Nueva York, 1979, p. 96 ss. 
definiciones de conceptos morales en términos de conceptos probablemente no-morales, y demostrar después, mediante procedimientos aceptados de sentido común y científicos, que las declaraciones así equiparadas con los juicios morales defendidos son las verdaderas [... ] Yo no he adoptado ninguna de estas concepciones de justificación [...] En realidad, he afirmado que estos principios (los morales) son contingentes en el sentido de que son elegidos en la situación original a la luz de hechos generales [... ] Por tanto, mejor será, en mi opinión, considerar una teoría moral exactamente igual que cualquier otra teoría, teniendo en cuenta sus aspectos socráticos. No hay razón para creer que sus primeros principios o supuestos necesiten ser autoevidentes, o que sus conceptos y criterios puedan ser sustituidos por otras nociones que puedan certificarse como no-morales." 9

\section{Justificación de los "derechos humanos"}

Cuando se pregunta por la justificación de los derechos humanos conviene precisar, para efectos de evitar confusiones en los planteamientos y obtener claridad en las respuestas, el universo de discurso en el cual se formulan las preguntas y se buscan las respuestas; es decir, se requiere indicar con exactitud el sistema de conocimiento, de acciones o de normas. Por otra parte, también es conveniente metodológicamente determinar con la mayor claridad posible el significado de las cuestiones.

Supongo que cuando se plantea el problema acerca de la justificación de los "derechos humanos", el universo de discurso no es el de un derecho positivo determinado, como el mexicano. Si en el derecho nacional, por ejemplo, se encuentran establecidos, definidos, determinados, otorgados los "derechos humanos", la justificación de los mismos no se encuentra en tal derecho positivo; está ausente de tal orden jurídico. Es preciso buscarla en otra parte. Algo similar ocurre con el concepto de significado; en efecto, el significante es la parte sensible, el símbolo o la expresión lingüística a la cual se pretende atribuir un significado; éste es la parte ausente de la expresión sensible y la significación es la relación entre los dos conceptos primeramente señalados. ${ }^{10}$

Pero ¿qué se encuentra en el derecho positivo?, ¿qué está presente en relación con los derechos humanos? Yo contestaría: las normas jurídicas en función de las conductas humanas y esa función integra uno o varios derechos humanos, si y sólo si se cumplen ciertas condiciones. Así, existe

9 John Rawls, Teoría de la justicia, Fondo de Cultura Económica, México/Madrid/Buenos Aires, 1979, pp. 638-639.

10 Oswald Ductor y Tzvetan Todorov, Dicionario enciclopédico de las ciencias del lenguaje, 7a. ed., Siglo Veintiuno Editores, México/España/Argentina/Colombia, 1981, p. 121 ss. 
un derecho humano cuando una norma de carácter constitucional regula la conducta de los particulares de tal forma que se establecen obligaciones o prohibiciones para las autoridades estatales y, en caso de inobservancia de las mismas o, lo que significa lo mismo, violación de derechos de los particulares, procede el juicio de amparo en defensa del particular, para anular el acto de autoridad o impedir, en su caso, que se aplique el acto inminente, y de esa manera recuperar la regularidad del sistema jurídico. Por ejemplo, el derecho a la vida.

El derecho a la vida, en el tema de la justificación, es lo que se pretende justificar y lo que lo justifica está ausente del derecho a la vida: el justificante está en otro lugar. Éste tiene alguna relación con lo que se pretende justificar: lo justificable y, en su caso, lo justificado. Al estar ausente, tiene una relación o se le busca una; "lo que se justifica" tendrá que ajustarse a algo definitivo.

Las instancias a las cuales se recurre cuando se pretenden justificar los "derechos humanos" son de varios tipos; así, valores, normas morales, normas religiosas, criterios, principios, hipótesis, la razón, "la naturaleza de las cosas", etcétera.

En la historia de los "derechos humanos" se han presentado diversos intentos filosóficos de justificación y ha sido, en general, la tentativa de responder a la pregunta acerca de la validez del derecho donde se enmarcan las inquietudes por ofrecer una justificación de los "derechos humanos". Las cuestiones planteadas han sido: ¿de dónde se deriva la validez del derecho?, ¿de dónde derivan su validez los "derechos humanos"? y, en particular, respecto del problema señalado, ¿de dónde deriva su validez la norma jurídica que establece el derecho a la vida?

En el ánimo del conocimiento, del pensar científico, la respuesta a las preguntas ha de ser satisfactoria. ${ }^{11}$

Una vez que se determina el universo de discurso para enfrentar el problema de la justificación, conviene precisar aún más las preguntas. En efecto, inquirir por la justificación de los "derechos humanos" es una exigencia muy general y la respuesta podría no aportar conocimientos por la tendencia a lo absoluto, en virtud de los términos de la pregunta.

Quizá no todos los llamados derechos humanos tengan la misma justificación en todos los órdenes jurídicos positivos. Convendrá formular mejor las preguntas con el fin de no perder especificidad en relación con algunos derechos y, en consecuencia, no tratarlos a todos con la misma medida, no ofrecer para todos la misma respuesta.

11 Karl Popper, "The Aim of Science", en Objective Knowledge. An Evolutionary Approach, Oxford at the Clarendon Press, Gran Bretaña, 1974. 
Con las ideas anteriores se busca una justificación relativa y, como cualquier intento, se ubicaría en el conocimiento científico y los avances siempre serían provisionales. Las clasificaciones y las jerarquizaciones, así como los criterios con base en los cuales se practican, constituyen un progreso en la forma de abordar el tema de la justificación. Así, también las relaciones entre los diversos niveles del lenguaje normativo coadyuvan en la aclaración de cómo se justifican los derechos humanos.

Podría preguntar por la justificación del "derecho a no ser molestado en la persona" previsto en el artículo 16 de la Constitución Federal de los Estados Unidos Mexicanos. Una primera aclaración semántica es sobre el significado de persona; a manera de aproximación podemos decir que se trata del sujeto de derecho, susceptible de tener otros derechos, y pasible de obligaciones. En principio, puede aludirse a características tales como la dignidad, la libertad y la igualdad.

En la proyección de "derechos humanos" las autoridades estatales tienen la obligación de no molestar a los gobernados. Un acto de molestia es una ofensa, cualquiera que sea su intensidad, en la esfera jurídica de los sujetos de derecho. En un régimen de derecho usualmente se establece el principio de facultades expresas, en virtud del cual las autoridades sólo pueden realizar las conductas expresamente autorizadas.

Respecto de otros ejemplos, como el derecho a la información, el derecho de los acusados a no ser compelidos a declarar en su contra y el derecho de toda persona a decidir de manera libre, responsable e informada sobre el número y el espaciamiento de sus hijos, las autoridades estatales tienen la obligación de informar, la prohibición de compeler a los acusados a declarar en su contra y la obligación de dejar que toda persona decida sobre el número y el espaciamiento de sus hijos.

Estos cuatro "derechos humanos" pueden justificarse a partir de la semántica respectiva y los valores relacionados con ella en un medio social histórico determinado; así, puede ser, respectivamente, procurar la adaptación al medio, la privacidad si se lo acusa de algún delito y el valor de la decisión sobre si se prolonga la carga genética.

Una vez establecida la semántica puede subsistir la pregunta por la justificación de la constitución. En las respuestas se puede llegar a la norma básica de derecho positivo que establece la validez de una constitución en tanto estatuye el procedimiento de reforma o adición de las demás normas constitucionales; se puede llegar también a hipótesis sobre la validez del derecho positivo determinado como la teoría de Hans Kelsen; a la regla de reconocimiento de Hart; o la hipótesis del contrato social de Rousseau, justificaciones de corte científico y formal, todas las cuales asumen la eficacia de los órdenes jurídicos de que se trate. 


\section{Violación de "derechos humanos" y justificación}

Otro problema surge cuando se presentan transgresiones de "derechos humanos" y se pretende su justificación. Pongamos por caso la hipótesis de que debe ser lo previsto en la constitución o, para los efectos que nos interesan, de que deben ser los "derechos humanos". En este sentido la cuestión que ha de plantearse es la siguiente: ¿es posible justificar la violación de derechos humanos en el universo de discurso de un derecho positivo determinado? La pregunta es acerca de las actuaciones de las autoridades estatales. En otras palabras: ¿el Estado puede privar de la vida, torturar, mentir o afectar la libertad de los gobernados? Quizá el contenido de cada una de las respuestas sea diferente en relación con cada derecho, aunque en ningún caso se justifiquen las transgresiones si continuamos en el mismo universo de discurso; en efecto, no es posible justificar una violación a los derechos humanos en el universo de discurso de la racionalidad que los otorga. Si el derecho humano es igual a $p$, entonces en la realidad puede darse no $p$, pero ello simplemente significa que se presenta un hecho en contra de lo previsto por la norma jurídica, pero no es posible justificar la transgresión. Si se tiene derecho a la vida como un derecho humano establecido en la constitución, la situación en que una autoridad prive de la vida a una persona puede ser caracterizada de dos maneras: o bien la autoridad tenía derecho a privar de la vida al individuo y en tal caso estaba cancelado previamente el derecho del particular a la vida, o bien la autoridad carecía de tal autorización y privar de la vida fue una transgresión que no se justifica.

Por ejemplo, cuando el Estado dicta a través de una sentencia la pena de muerte y la ejecuta, el Estado tiene autorización para privar de la vida y el particular, en esa relación, carece del derecho a la vida. Se cancela el derecho humano del individuo en virtud de alguna razón poderosa en favor del Estado que justifica su acción, mas no puede afirmarse que se trate de justificar una violación a los derechos humanos. Se trata de una situación vista con enfoques diferentes; en el último de ellos se presentan argumentos o diferencias que neutralizan, cancelan o derogan la primera de las situaciones en que se tiene un derecho humano.

El Estado está justificado en sus acciones cuando se encuentra autorizado expresamente por las normas constitucionales para realizar tales acciones. Una justificación bastante flexible es la existencia de facultades discrecionales, porque conforme a su ejercicio tiene competencia para realizar la conducta $p$ y la contraria, esto es, no $p$, porque al realizarse cualquiera de ellas se cumple formalmente con la norma constitucional. Hay otras situaciones en las cuales se lo faculta, por ejemplo, a actuar, atendiendo a la "utilidad pública". En estos casos y similares difícilmente podría 
afirmarse transgresión a derechos humanos y menos hablar de la justificación de la "transgresión" a los mismos. Un problema de interpretación jurídica y de política legislativa se presenta cuando se incorpora gran número de expresiones de este tipo, pues son facilidades exageradas para que las autoridades estatales justifiquen sus actuaciones en las normas constitucionales, situaciones en las que se, restringe considerablemente la acción de los particulares en el ámbito de sus derechos frente al Estado.

Han sido evidentes los casos de tortura, o sea de transgresión al derecho humano de los acusados a no ser compelidos a declarar en su contra; y no aparece, no surge, no se vislumbra ninguna autorización constitucional para torturar ni, en consecuencia, justificación alguna. No se advierte en la situación descrita "esa instancia independiente" que justifique la acción de las autoridades. Ésta tendrá que superar la paradoja o la contradicción de "para cumplir con el derecho puedo violarlo", y no es posible tal superación si estamos involucrados en el juego lingüístico o forma de vida de un derecho positivo determinado.

Sobre el principio de que el derecho es obligatorio para las autoridades no aparece, pues, forma de justificar una violación de derechos humanos. Tal principio forma parte del sistema de subsistencia de la sociedad organizada jurídicamente, con especial referencia a los aspectos políticos y, en particular, al régimen democrático.

Cuando se empiezan a usar expresiones como "el interés público", "la moral pública", "la utilidad pública", "la paz pública", mencionadas en los preceptos constitucionales como límite a los "derechos humanos", se acercan peligrosamente las "justificaciones" estatales al empleo de frases similares no incluidas en la constitución, tales como "la República lo exige", o, lo que es más grave, "por razón de Estado". Una razón de Estado es ajena al orden jurídico; desempeña en un nivel diferente, en otra racionalidad, y tomar una decisión con base en ella es desdeñar la razón para actuar ofrecida por el derecho, independientemente del contenido de la razón del Estado. Superar la razón del derecho es transgedirlo. En una situación conflictiva, si se ponderaron los argumentos contenidos en las "razones para actuar" se da peso de razón absoluta a la "razón de Estado". "Es la ragione di Stato de Maquiavelo — sostiene Kelsen - la célebre 'razón de Estado', cuyo nombre encubriría la imposición de todo completo de postulados políticos favorables al príncipe y sus secuaces, frente al orden jurídico en vigor." ${ }^{12}$

Cuando se apela a estas últimas instancias se presume un exceso a las disposiciones constitucionales; las actuaciones, sean decisiones o acciones, van más allá de lo jurídico y de sus fundamentos. Una "razón de Estado" en

12 Hans Kelsen, Teoría general del Estado, Editora Nacional, México, 1959, pp. 118-119. 
manos de quienes ejercen el poder es ilimitada; todo "lo justifica", incluso las violaciones constitucionales de algunos de los "derechos humanos".

Las dos manifestaciones, casos eventuales y casos generalizados, son fácilmente descriptibles y controlables intersubjetivamente por quienes tienen interés en el desarrollo de la vida política. Son controlables, desde el punto de vista del conocimiento, por un grupo más o menos amplio de comunidades científicas, de partidos, de profesionales, de universitarios y, en general, de gobernados que tienen diferentes ámbitos de acción. Las consecuencias sociales de estos controles son diferentes, según sea el grado de involucramiento, oportunidad y calidad de argumentación.

Las consecuencias no están reglamentadas y, por lo tanto, no existe una ideología positiva a la cual referirse para sustentar la pertinencia de que se produzca algún tipo de ellas; se trata de opiniones y de acciones de los grupos influyentes que cumplen ciertos papeles sociales, mas carecen de poder y de la organización adecuada para oponerse por medios legales de defensa a las decisiones de las autoridades estatales. Sus controles intersubjetivos están privados de consecuencias jurídicas. En esta perspectiva me refiero a las manifestaciones de protesta bien fundadas que pueden ir desde opiniones a acciones como marchas, plantones, resistencia civil y actos de desobediencia civil. En todas ellas lo decisivo es la presentación de argumentos en contra de la "violación" de derechos humanos.

\section{Justificación satisfactoria}

Desde el punto de vista teórico, la justificación ha de ser satisfactoria, como toda explicación de la empresa científica y de la acción humana. La satisfactoriedad alude al cumplimiento de ciertos requisitos en el desarrollo de la actividad para justificar algo, así como sus resultados.

Señalaría como los requisitos mínimos de toda justificación los siguientes:

4.1. Informativa. De acuerdo con esta nota, una justificación es una explicación argumentada y sistematizada de variables de distinto tipo, suficiente para cumplir con los propósitos de la comunicación, es decir, ofrecer elementos para adaptarse a un medio determinado; asi, ha de ser verdadera, completa y oportuna. Esta característica se opone a las justificaciones triviales en el sentido de circularidad, porque en éstas no se advierte la intención de presentar más y mejores argumentos y se aproxima a las actitudes dog. máticas.

4.2. Pública. Las explicaciones han de ser sometidas a la opinión de las diversas comunidades interesadas a través de los medios de comunicación idóneos, de tal manera que, en principio, exista testimonio indubitable de la 
justificación y cualquier persona pueda tener acceso a los datos respectivos para su análisis y toma de posición. En tal virtud la justificación no puede ser privada ni secreta.

4.3. Objetiva. Las explicaciones han de ser convincentes en el marco de referencia de que se trata, principalmente en el legal, en el político y en los hechos sociales relacionados. Se exige, en virtud de esta característica, un control intersubjetivo de las razones expuestas, esto es, de "lo que justifica"; así, no sería adecuado apelar a los "estados mentales" para sustentar una justificación. Si se argumenta que se tomó una decisión porque se tuvo la inspiración de que era lo mejor, tal justificación no parece ser objetiva. Las teorías intuicionistas y sus "referencias" no son adecuadas para ofrecer argumentos o explicaciones susceptibles de control intersubjetivo o de ser refutadas. ${ }^{13}$ Serían, parafraseando a Gaston Bachelard, un obstáculo epistemológico, y superarlo prácticamente implica no aludir a ellas. ${ }^{14}$

Las justificaciones subjetivas ni se verifican ni se refutan. Como el anhelo de conocer objetivamente está presente en el tema de la justificación, el primer tipo de justificaciones no son útiles en estos intentos.

4.4. Consistente. En todo caso, los enunciados en los cuales se presenta una justificación no deben contener premisas contradictorias, y las conclusiones han de seguirse de las premisas.

Los principios lógicos de no contradicción y los del silogismo han de respetarse en el discurso a través del cual se presenta una justificación.

4.5. Exacta. Conforme a la característica de la exactitud se ha de advertir la adecuación entre lo justificado y lo que justifica. La comparación, la medición y los juicios sobre la adecuación son actividades intelectuales nacesarias cuando se presenta una justificación, como relación entre lo justificado y lo que justifica.

4.6. Relativa. Esta característica recoge uno de los atributos en el desarrollo del conocimiento científico; en efecto, en la base del progreso se encuentra la posibilidad de que la ciencia avanza y no obtiene un conocimiento definitivo y absoluto sino provisional y susceptible de ser superado en virtud de nuevos problemas surgidos a partir de descubrimientos, aunque también mediante la formulación de mejores explicaciones elaboradas sin la presencia de aquéllos.

13 Cfr. Felix E. Oppenheim, Los principios morales en la filosofía politica, Fondo de Cultura Económica, México, 1975 (Breviarios), p. 69 ss.

14 Gaston Bachelard, La formación del espíritu científico. Contribución a un psicoanálisis del conocimiento objetivo, 8a. ed., Siglo Veintiuno Editores, México/España/Colombia, 1979, p. 15 ss., p. 115 ss. 
4.7. Externa. Esta característica exige recurrir a la última instancia independiente que justifica en el universo de discurso planteado, para así tener la visión completa de la justificación y no inquirir incesantemente por otra instancia. Se presenta en ciertos ámbitos o instancias que justifican y que requieren ser justificadas, y así en varios momentos sucesivos. En estos casos se trata de una justificación interna, mas no responde a la exigencia teórica fundamental de justificar.

Las anteriores son características centrales de un concepto abierto de justificación satisfactoria; este aserto implica la posibilidad de señalar otras, como la sencillez, el amplio alcance y el carácter fructífero, las cuales se relacionan con el conocimiento científico. ${ }^{15}$

\section{La satisfactoriedad en la justificación de los "derechos humanos"}

Una justificación satisfactoria de los "derechos humanos", en general, y de cada "derecho humano", en particular, ha de cumplir los requisitos mínimos referidos en el apartado anterior; es decir, ha de ser informativa, pública, objetiva, consistente, exacta, relativa y externa.

Conviene precisar los niveles en los cuales puede exigirse una justificación. Se dijo, al citar a Sissela Bok, que la justificación requiere un público; esta orientación es útil para la precisión exigida, aun cuando en ocasiones no se advierta claramente cuál es ese público o públicos o aun cuando haya intersecciones de públicos, incluso con racionalidades contradictorias.

Las autoridades tienen que justificar sus decisiones y acciones ante distintos públicos, como las diversas comunidades científicas, la opinión pública, otras autoridades estatales constituidas, los partidos a los que éstas pertenezcan o los de oposición. Hay un vínculo entre la obligación de responder de las autoridades y la justificación. No es ante sus conciencias, éstas son estados mentales.

Las mismas exigencias se dan para las disciplinas que estudian los derechos humanos, principalmente la ciencia jurídica, la política y la ética, las cuales ofrecen las justificaciones respectivas. En política legislativa, es decir, la disciplina que se relaciona con los problemas de creación o modificación de leyes, el conjunto de derechos del individuo o de grupos frente a las autoridades estatales y la exigencia de respetarlos son variables siempre presentes.

Actualmente se plantea, a propósito de problemas relacionados con narcotráfico - los cuales, se asume, producen un elevado costo social-, la

15 Thomas Kuhn, The Essential Tension. Selected Studies in Scientific Tradition and Change, The University of Chicago Press, Chicago/Londres, 1977; principalmente cfr. el artículo "Objectivity, Value Judgment and Theory Choice". 
posibilidad de efectuar reformas constitucionales y legales, como serían, en situaciones excepcionales, la de considerar delictiva la intención de cometer un delito (distinta a la tentativa de realizarlo) y la de permitir el espionaje telefónico 0 a través de otras comunicaciones. ${ }^{16}$

Sería pertinente someter ambas propuestas a la prueba de si poseen una justificación satisfactoria en términos de los derechos humanos relacionados con la privacidad de las personas y con la seguridad jurídica y el principio de legalidad previstos en los artículos 14 y 16 constitucionales.

\section{Justificación en el pensamiento de Javier Esquivel (un sentido recuerdo filosófico) ${ }^{17}$}

Cuando Javier Esquivel aborda el tema de la justificación, desde el punto de vista del lenguaje, inquiere por respuestas a un nivel diferente de aquel en que encuentra lo que pretende justificar. Se trata de una remisión a algo diferente.

Expone el papel de la justificación de manera muy precisa cuando analiza el tema del tiranicidio, en donde proporciona algunas consideraciones para la posible justificación moral de este tipo de homicidio. También sostiene: "la imagen que me propongo construir es la del tiranicidio como un asesinato político que parece prima facie justificado moralmente", y anuncia que presentará el núcleo de la justificación moral de esta acción.

En particular, conviene indicar los argumentos que Javier Esquivel presenta para justificar el tiranicidio, entendido como "un acto intencional por el cual una figura política es privada de la virla por razones políticas y en forma legal". Entre las razones alude a que "la víctima sea responsable de varios crimenes en contra de sus súbditos, en particular en contra de sus

16 Cfr. Proceso, no. 868, 21 de junio de 1993, pp. 14-17.

A propósito del valor de la regla y de las excepciones es pertinente aludir a los siguiente parágrafos de las Investigaciones filosóficas, de Ludwig Wittgenstein. "142 [... ] Y si las cosas fueran totalmente distintas de como efectivamente son — si no hubiera, por ejemplo, ninguna expresión característica de dolor, de miedo, de alegria; si la regla se convirtiera en excepción y la excepción en regla; o si ambas se convirtieran en fenómenos de aproximadamente la misma frecuencia- entonces nuestros juegos de lenguaje normales perderían con ello su quid." "199 [... ] una regla no podía determinar ningún curso de acción porque todo curso de acción puede hacerse concordar con la regla; $[.$. ] si todo puede hacerse concordar con la regla, entonces también puede hacerse discordar; en consecuencia, no habrá ni concordancia ni desacuerdo". Para una mayor explicación y aplicación de estas ideas se puede consultar Agustín Pérez Carrillo, "Autoridad de la Constitución (notas sobre una forma de vida)", Alegatos (Universidad Autónoma Metropolitana, División de Ciencias Sociales y Humanidades, México), no. 22, septiembre-diciembre, 1992.

17 Las citas textuales son tomadas del artículo de Javier Esquivel Pérez, "Assassination and Tyrannicide", Crítica. Revista Hispanoamericana de Filosofía (México), vol. XI, no. 33, diciembre, 1979. 
derechos a la vida, a la libertad y a la igualdad". Señala seis características de la situación en la cual se puede justificar y, en atención a la determinación de la ausencia o presencia de las mismas, se puede concluir si en algún caso particular existe justificación de privar de la vida a una persona.

En relación con la justificación del tiranicidio, recurre a un modelo que reúne las siguientes características: 1 ) existencia de motivos morales para hacerlo; 2) éxito en la empresa; 3) existencia de buenas razones para creer en el éxito (en este sentido las consecuencias desempeñan un papel importante en la justificación); 4) tratarse del último recurso para resolver la situación sociopolítica; 5) que la víctima sea un tirano, y6) que se seleccione el medio menos doloroso y más rápido para lograr la privación de la vida. Como consecuencia de las ideas anteriores, se puede derivar que un caso justificado de tiranicidio sería aquel que tiene las características y satisface las condiciones descritas anteriormente. "Una acción, en consecuencia, que estaba originalmente prohibida será justificada si uno es capaz de señalar las circunstancias que nos capacitan su deducción de una regla más específica que lo permita, o aún vincula a su realización."

La exigencia de justificar, en el pensamiento de Javier Esquivel, es una muestra de su actitud crítica y del valor de no asumir dogmas en la empresa filosófica, científica o moral, así como una vía adecuada para evitar colapsos en la comunicación con los integrantes de los diversos grupos involucrados en la comprensión y solución de problemas similares. 